\title{
Craving in Alcohol-Dependent Patients After Detoxification Is Related to Glutamatergic Dysfunction in the Nucleus Accumbens and the Anterior Cingulate Cortex
}

\author{
Jochen Bauer',5, Anya Pedersen 2,5 , Norbert Scherbaum³, Johanna Bening², Johanna Patschke', \\ Harald Kugel ${ }^{4}$, Walter Heindel ${ }^{4}$, Volker Arolt ${ }^{1}$ and Patricia Ohrmann*,1 \\ 'Department of Psychiatry, University of Muenster, Muenster, Germany; '2Department of Psychology, University of Muenster, Muenster, \\ Germany; ${ }^{3}$ Addiction Research Group, Department of Psychiatry and Psychotherapy, LVR-Hospital Essen, University of Duisburg-Essen, \\ Essen, Germany; ${ }^{4}$ Institute of Clinical Radiology, University of Muenster, Muenster, Germany
}

\begin{abstract}
The upregulation of glutamatergic excitatory neurotransmission is thought to be partly responsible for the acute withdrawal symptoms and craving experienced by alcohol-dependent patients. Most physiological evidence supporting this hypothesis is based on data from animal studies. In addition, clinical data show that GABAergic and anti-glutamatergic drugs ameliorate withdrawal symptoms, offering indirect evidence indicative of glutamatergic hyperexcitability in alcohol-dependent subjects. We used proton magnetic resonance spectroscopy to quantify the glutamate (Glu) levels in healthy control subjects and in alcohol-dependent patients immediately after detoxification. The volumes of interest were located in the nucleus accumbens (NAcc) and the anterior cingulate cortex (ACC), which are two brain areas that have important functions in reward circuitry. In addition to Glu, we quantified the levels of combined Glu and glutamine (Gln), N-acetylaspartate, choline-containing compounds, and creatine. The Glu levels in the NAcc were significantly higher in patients than in controls. Craving, which was measured using the Obsessive Compulsive Drinking Scale, correlated positively with levels of combined Glu and Gln in the NAcc and in the ACC. The levels of all other metabolites were not significantly different between patients and controls. The increased Glu levels in the NAcc in alcohol-dependent patients shortly after detoxification confirm the animal data and suggest that striatal glutamatergic dysfunction is related to ethanol withdrawal. The positive correlation between craving and glutamatergic metabolism in both key reward circuitry areas support the hypothesis that the glutamatergic system has an important role in the later course of alcohol dependence with respect to abstinence and relapse.

Neuropsychopharmacology (20 I3) 38, I40 I-I408; doi:I0.1038/npp.2013.45; published online I3 March 2013
\end{abstract}

Keywords: alcoholism; glutamate; nucleus accumbens; craving; 'H MRS; withdrawal

\section{INTRODUCTION}

The pathophysiology of acute ethanol withdrawal symptoms and craving is thought to be closely related to cerebral glutamatergic dysregulation. The use of experimental microdialysis in animal studies has consistently demonstrated that there are significant increases in extracellular glutamate $(\mathrm{Glu})$ related to consecutive cycles of intoxication and withdrawal (Rossetti et al, 1999; Gu et al, 2012). In addition, several animal studies have shown that acamprosate, a taurine homolog and Glu antagonist, reduces extracellular cerebral Glu levels (De Witte, 2004; Hinton et al, 2012). The increased Glu release during the initial

*Correspondence: Dr P Ohrmann, Department of Psychiatry, University of Muenster, Albert-Schweitzer Campus I, A9, Muenster 48|49, Germany, Tel: + 0049 25I 8356645;

Fax: + 0049 25। 8356612; E-mail: ohrmann@uni-muenster.de

${ }^{5}$ These authors contributed equally to this work.

Received 3 November 2012; revised 6 February 2013; accepted 6 February 2013; accepted article preview online 12 February 2013 stages of withdrawal is thought to be the result of changes in the sensitivity of $N$-methyl-D-aspartic acid (NMDA) receptor subunits, because NMDA receptor upregulation in the brain's reward circuitry has been observed in alcoholic patients (Siggins et al, 2003; Ward et al, 2009). Pharmacological intervention studies focused on alcohol-dependent patients indirectly support the hypothesis that Glu levels are elevated during acute withdrawal because GABAergic and anti-glutamatergic compounds have been found to be effective in reducing withdrawal symptoms (Krupitsky et al, 2007). Acamprosate has also been shown to prevent relapse in individuals with chronic alcoholism (Roesner et al, 2008). However, few in vivo studies have explored the role of glutamatergic dysfunction in alcohol-dependent patients (Gass and Olive, 2008).

Single-voxel proton magnetic resonance spectroscopy $\left({ }^{1} \mathrm{H}\right.$ MRS) allows for the noninvasive in vivo measurement of Glu concentrations within selected areas of the brain. Whereas at $1.5 \mathrm{~T}$, Glu and glutamine (Gln) are not distinguishable and are usually reported as a combination of Glu and Gln (Glx), studies using 3-T scanners with 
optimized acquisition parameters have produced increasingly reliable and distinct Glu signals (Hancu, 2009; Mullins et al, 2008; Wijtenburg and Knight-Scott, 2011).

Due to the methodological shortcomings inherent in measuring Glu levels, previous $1.5 \mathrm{~T}$ spectroscopy studies have focused on the more reliable quantification of $\mathrm{N}$ acetylaspartate (NAA), a marker of neuronal integrity, and of choline-containing compounds (Cho), creatine and phosphocreatine (Cr), and myo-inositol (mI). In most but not all studies, reduced levels of NAA and increased levels of $\mathrm{mI}$ in different brain areas were noted in alcohol-dependent patients, and these levels tended to normalize during periods of abstinence (Licata and Renshaw, 2010; Mason et al, 2005). Chronic alcoholism has also been shown to be associated with a decreased signal for Cho, especially in the frontal lobes, whereas an increase in Cho was noted in patients during periods of abstinence (Modi et al, 2011; Ende et al, 2005). Changes in the levels of NAA, Cho, and mI are thought to be due to the direct and indirect toxic effects of chronic ethanol use, which result in consecutive gray and white matter damage and wide-spread cerebral volume reductions in patients with chronic alcoholism (Fortier et al, 2011).

Few spectroscopy studies have focused on glutamatergic metabolism in patients with alcoholism. Most of these studies have investigated the anterior cingulate cortex (ACC) and have yielded mixed results. Lee et al (2007) found no significant differences in the Glu levels in the ACC and insula in patients compared with healthy controls after 2 weeks of abstinence, whereas Umhau et al (2010) reported a significant decrease in the Glu level in the ACC of alcoholdependent patients after a 4-week trial of acamprosate relative to the Glu level for the group treated with a placebo. Hermann et al (2012) described an increase in the Glu level that was directly related to the emergence of withdrawal symptoms in alcohol-dependent patients; this increase was followed by a significant decrease within 2 weeks of pharmacologically supported detoxification. Thoma et al (2011) studied alcohol-dependent patients who either had been abstinent for 1 year or were still drinking, and they reported decreased Glu levels and higher Gln levels in the ACC in both patient groups relative to healthy controls.

In addition to dopaminergic neurotransmission, glutamatergic neurotransmission has an important role in motivation, learning and memory, and glutamatergic projections and/or neurons expressing Glu receptors are widely distributed in the mesolimbic reward circuitry (Kelley, 2004). These excitatory glutamatergic innervations are the physiological basis for the dopamine-Glu interactions that regulate addiction-related synaptic plasticity (Omelchenko et al, 2007; Groenewegen et al, 1999). Neuroanatomically, activation of the mesocorticolimbic system, including the ventral tegmental area (VTA), nucleus accumbens (NAcc), amygdala, and prefrontal cortex, constitutes the pathway by which alcohol and drugs are thought to mediate their reinforcing effects. In particular, the NAcc has been identified as an important site for the acquisition and expression of addictive behavior (Kalivas and Volkow, 2011). Glutamatergic inputs from the ACC, amygdala, and hippocampus as well as dopaminergic inputs from the VTA in the brainstem converge upon the NAcc. The rostral and dorsal ACC project directly into the NAcc shell and core region, respectively.
In our study, we used ${ }^{1} \mathrm{H}$ MRS to investigate the NAcc and the ACC, two areas that are essential to the brain's reward circuitry. We hypothesized that these brain areas would be affected by the glutamatergic pathophysiology associated with alcohol withdrawal. In addition, we hypothesized that there would be reduced NAA levels in the ACC due to the neurotoxicity associated with chronic alcohol consumption, leading to neuronal damage, especially in the frontal and temporal lobes (Fortier et al, 2011).

\section{MATERIALS AND METHODS}

\section{Participants}

We recruited 29 alcohol-dependent patients who were admitted for inpatient detoxification to the Department of Psychiatry at the University of Muenster and the Westphalia Psychiatric Clinic in Muenster. We also recruited 31 healthy participants using advertisements in local newspapers. All patients fulfilled the DSM-IV criteria for alcohol dependence (SCID, Structured Clinical Interview for DSM-IV). All participants were male and right handed (Edinburgh Handedness Inventory). The exclusion criteria for all participants included are as follows: current psychiatric disorders, neurological disorders, serious head injury, liver cirrhosis, acute pancreatitis, and the abuse of or dependence on any substance other than nicotine (both groups) or alcohol (alcohol-dependent group). We used the Alcohol Use Disorder Identification Test (Saunders et al, 1993) and the SCID interview to exclude all healthy subjects with alcohol abuse or dependence. The European Addiction Severity Index (EuropASI; Gsellhofer et al, 1994) was implemented in the patient group to assess the clinical characteristics of the subjects' alcohol dependence. Obsessions and compulsions related to craving and drinking behavior were assessed using the German version of the Obsessive Compulsive Drinking Scale (OCDS-G; Anton et al, 1995, 1996). The scale is a 14-item self-rated OCDS measure of the obsessive and compulsive aspects of craving, eg, drinking-related thoughts, urges to drink, and the ability to resist those thoughts and urges. The subscores 'obsessions' and 'compulsive drinking' were calculated based on the methodology proposed by Anton et al $(1995,1996)$. The demographic and clinical characteristics of the patients and controls are summarized in Table 1.

To obtain high-quality spectroscopy data, subjects were required to lie motionless in the scanner for approximately $1 \mathrm{~h}$. Withdrawal symptomatology includes a variety of physical symptoms, such as tremors, sweating, nausea, and headache that make it challenging to remain motionless. Therefore, we decided to investigate patients after acute detoxification, although one would expect the Glu level to be the highest during the acute withdrawal period. All patients were investigated within 10 days of admission to the hospital and at least $24 \mathrm{~h}$ after termination of a standardized medication for withdrawal symptomatology based on the guidelines of the German Society for Addiction Research and Therapy (Mundle et al, 2006). Symptomtriggered doses of either diazepam or clomethiazole were administered to ameliorate severe symptomatology and were consecutively reduced according to a fixed dosing reduction regimen. In our study, 8 patients were treated 
Table I Demographic and Clinical Characteristics of Alcohol-Dependent Patients and Healthy Controls

\begin{tabular}{|c|c|c|c|c|}
\hline & Patients $(n=29)($ mean (SD)) & Controls $(n=3 I)($ mean (SD)) & Statistics & $p$ \\
\hline Age (years) & $40.2(7.5)$ & $36.6(10.1)$ & $t(58)=-1.537$ & 0.130 \\
\hline School education (years) & $10.7(1.8)$ & $11.4(1.50)$ & $t(58)=1.709$ & 0.093 \\
\hline Ethanol/day ( $\mathrm{g}$; mean of last 30 days) & $300.7(177.6)$ & $7.2(4.6)$ & $t(58)=-7.967$ & 0.001 \\
\hline Duration of alcohol dependence (years) & $22.6(7.8)$ & - & - & - \\
\hline Age at first intoxication (years) & | $8 . \mid(4.5)$ & - & - & - \\
\hline Nicotine (yes: no) & 27: 2 & 5: 26 & $x^{2}=35.669$ & 0.001 \\
\hline Cigarettes/day (smokers only) & $25.2(12.2)$ & $4.4(3.9)$ & $t(58)=-3.747$ & 0.001 \\
\hline
\end{tabular}

Three subjects did not complete the OCDS.

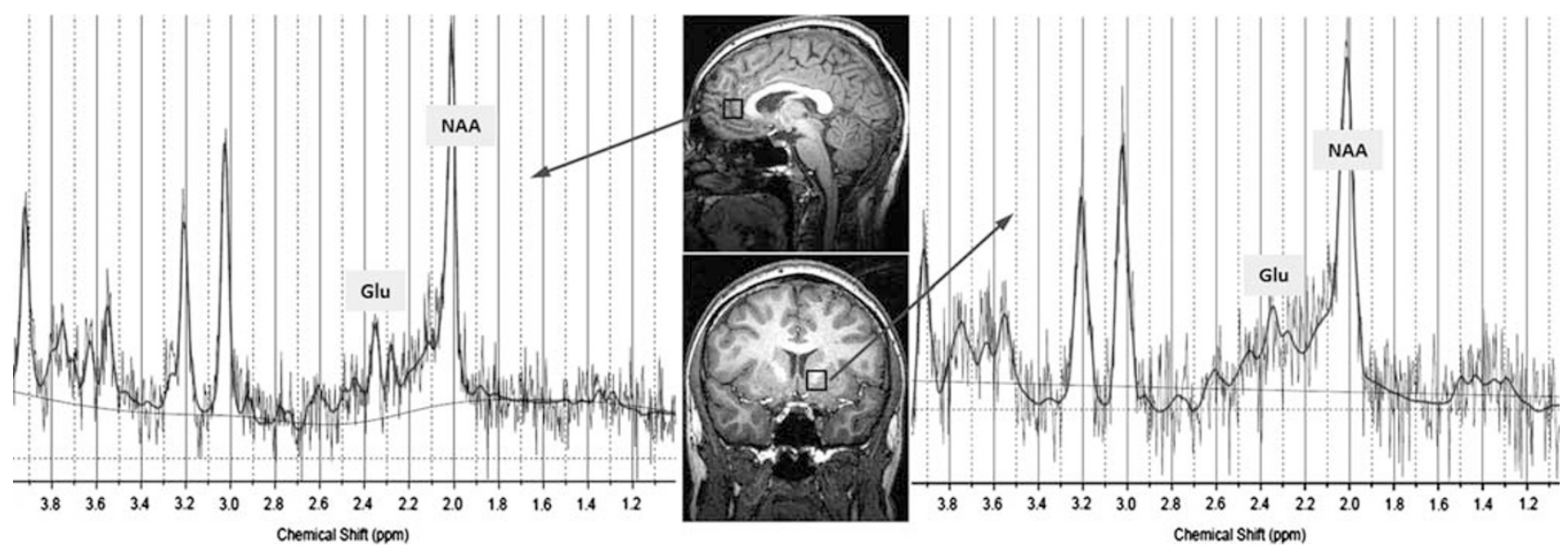

Figure I VOI locations in the bilateral rostral ACC (top) and in the left NAcc (bottom) with representative spectra in an alcohol-dependent patient.

with clomethiazole, 12 patients were treated with diazepam, and 9 patients did not receive any medication. This study was approved by the local ethics committee. After a complete description of the study to all participants, written informed consent was obtained. All participants received financial compensation of $20 €$.

\section{${ }^{1} \mathrm{H}$ MRS}

All subjects were scanned in a 3-T scanner (Gyroscan Intera 3 T, Philips Medical Systems, Best, The Netherlands) with a transmit/receive head coil. T1-weighted 3D high-resolution anatomical images of the whole brain were acquired using a 3D fast gradient echo sequence ('Turbo Field Echo'): $\mathrm{TR}=7.5 \mathrm{~ms}$, TE $3.4 \mathrm{~ms}, \mathrm{FA}=9^{\circ}$, two signal averages, and an inversion prepulse every $814.5 \mathrm{~ms}$. These images were reconstructed into cubic voxels with a $0.5-\mathrm{mm}$ edge length. From this data set, slices in three orthogonal planes were displayed using multiplanar reconstruction for localization of the spectroscopic volumes of interest (VOIs; $15 \mathrm{~mm} \times 15$ $\mathrm{mm} \times 15 \mathrm{~mm}$ ). The voxel position in the ACC was chosen to cover the bilateral rostral ACC (Brodmann area 24). Axially, the voxel was placed close to the most anterior part of the genu of the corpus callosum, with the cingulate sulcus as the anterior border. In the sagittal slices, the inferior border of the VOI was placed parallel to the line connecting the anterior and posterior commissures (Figure 1). The NAcc is defined as the ventral part of the striatum. Thus, the VOI was placed in such a way as to cover the most ventral part of the striatum in the coronal and sagittal slices with the ventral corner of the lateral ventricle as a topographic marker point (Figure 1). The size of our cubic VOI is approximately $3.4 \mathrm{~cm}^{3}$; therefore, in addition to the ventral striatum, white matter belonging to the internal capsule was also included in our VOI. In both VOIs, the amounts of gray and white matter did not differ significantly between patients and controls.

Spectral data were acquired with a point-resolved spectroscopy sequence using the following parameters: $\mathrm{TE}=32 \mathrm{~ms}$, $\mathrm{TR}=2000 \mathrm{~ms}$, bandwidth $=2000 \mathrm{~Hz}, 2048$ data points, and 128 signal averages. A short echo time was chosen to obtain the optimal selectivity for Glu and sufficient quality for Glu and Gln (Hancu, 2009; Mullins et al, 2008). Example spectra for both regions of interest are presented in Figure 1. For quality control, a phantom with a concentration of $50 \mathrm{mM}$ creatine was measured before each MRS session using the standard study protocol. The overall scanner variance caused by external factors during the acquisition of all data was $1.7 \%$. Quantification of the spectra was based on LCModel spectral fitting (Provencher, 1993). The concentra- 
Table 2 Means and Standard Deviations of the Metabolite Concentrations in the NAcc (IU) and Group Comparisons (T Test for Independent Samples; Bonferroni Correction: $P<0.005)$

\begin{tabular}{|c|c|c|c|c|}
\hline & Patients $(n=29)^{\text {a }}($ mean $(S D))$ & Controls $(n=3 \mathrm{I})^{\mathrm{b}}($ mean (SD) $)$ & Statistics & $p$ \\
\hline Glu (IU) & $10.17(1.14)$ & $9.06(1.34)$ & $t(45)=3.045$ & 0.004 \\
\hline Cho (IU) & $1.90(0.30)$ & $1.97(0.36)$ & $t(56)=-0.825$ & 0.413 \\
\hline $\operatorname{Cr}(I U)$ & $7.28(0.79)$ & $7.40(0.65)$ & $t(57)=-0.625$ & 0.534 \\
\hline
\end{tabular}

IU: Institutional units.

aAA $n=29$, Glu $n=22$, Glx $n=27$, Cho $n=28$, and $\operatorname{Cr} n=29$.

${ }^{\mathrm{b}} \mathrm{NAA} n=30$, Glu $n=25$, Glx $n=28$, Cho $n=30$, and $\mathrm{Cr} n=30$.

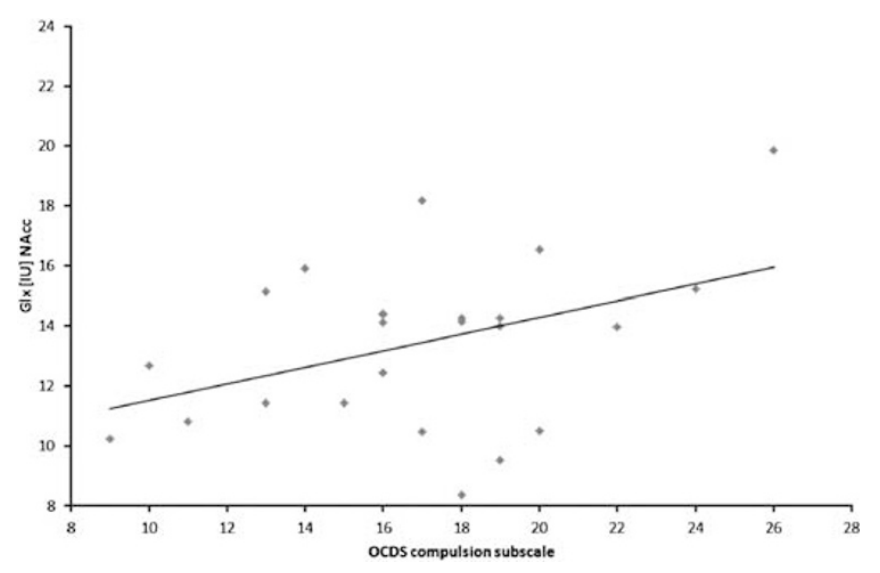

Figure 2 Correlation between the compulsion subscale of the Obsessive Compulsive Drinking Scale (OCDS) and the combined glutamate and glutamine (Glx) level in the nucleus accumbens (NAcc).

tion values were normalized to the unsuppressed water signal from the same VOI and are expressed in institutional units. Only metabolite concentrations with Cramér-Rao lower bounds (CRLBs) below 20\% were accepted and used for the following analyses. The CRLBs for all metabolites were not significantly different between patients and controls, the mean CRLBs for Glu were below 14\% for both groups. To correct the metabolite concentrations for CSF, a procedure developed in-house based on the VBM5 algorithm (VBM5, http://dbm.neuro.uni-jena.de/vbm/) was used to segment the T1-weighted data sets.

\section{Statistics}

SPSS was used for the data analysis (SPSS 18.0 for windows, SPSS, Inc., Chicago, Illinois). The metabolite levels and the clinical and demographical variables were analyzed to identify between-group differences using the unpaired $t$-test for independent samples, ANOVA (effect of medication), and Pearson's $X^{2}$. The data distributions were tested for normality using P-P plots and the Kolmogorov-Smirnov test. Pearson's correlation coefficients were computed to determine the correlations between metabolite levels and clinical characteristics. The number of smokers differed significantly between the two groups. Therefore, this variable could not be used as a covariate in an ANCOVA to analyze the effects of smoking on metabolites (cp. Miller and Chapman, 2001). Instead, partial correlations adjusting for the number of cigarettes smoked per day were computed. Partial correlations were also performed for gray matter fractions in both VOIs. Significance was assumed for $p$ values $<0.05$ (two-tailed). A Bonferroni correction was used for comparisons of the metabolite concentrations $(p<0.005$; 10 comparisons (5 metabolites and 2 voxels)).

\section{RESULTS}

\section{${ }^{1} \mathrm{H}-\mathrm{MR}$ Spectroscopic Analysis of the NAcc}

The mean metabolite concentrations in the NAcc are displayed in Table 2. The group comparisons revealed significantly higher Glu concentrations in the NAcc in the patients relative to the controls $(t(45)=-3.045, p=0.004)$. None of the other metabolites differed significantly between the two groups (all $p$-values $>0.05$; Table 2 ). There were no significant differences between the non-medicated patients and the patients medicated with either diazepam or clomethiazole (all $p$-values $>0.05$ ). In addition, there was no significant difference in the Glu concentration between patients treated with diazepam and those treated with clomethiazole $(t(17)=-1.437, p=0.169)$. In our patients, the Glx concentrations in the NAcc correlated positively with craving (total OCDS score; $r=0.439, p=0.036$ ), ie, patients with higher Glx levels reported more craving. This positive relationship was noted for the compulsion OCDS subscale $(r=0.412, p=0.045$, Figure 2; partial Spearman's $r=0.459, p=0.036$, controlling for the number of cigarettes smoked per day; partial Spearman's $r=0.401, p=0.072$, controlling for gray matter), but not for the obsession subscale $(r=0.324, \quad p=0.114)$. Separate statistical analysis of estimated Gln concentrations, calculated by subtracting Glu from Glx, revealed that craving was significantly correlated to Gln $(r=0.563, p=0.010$; partial Spearman's $r=0.491, p=0.033$, controlling for the number of cigarettes smoked per day; partial Spearman's $r=0.542, p=0.016$, controlling for gray matter). There were no significant correlations between the metabolite levels and the demographic characteristics, and there were no significant correlations between the severity of withdrawal, as measured by the cumulative doses of diazepam or clomethiazole, and the metabolite levels. In our patients, there was no significant correlation between the metabolite levels and the number of cigarettes smoked per day. 
Table 3 Means and Standard Deviations of the Metabolite Concentrations (IU) in the ACC and Group Comparisons ( $T$ Test for Independent Samples; Bonferroni Correction: $p<0.005$ )

\begin{tabular}{|c|c|c|c|c|}
\hline & Patients $(n=29)^{\text {a }}($ mean (SD)) & Controls $(n=3 I)^{\mathbf{b}}($ mean (SD)) & Statistics & $\mathbf{p}$ \\
\hline NAA (IU) & $10.86(1.20)$ & $10.93(0.92)$ & $t(56)=-0.125$ & 0.901 \\
\hline Glu (IU) & $12.02(1.50)$ & $11.91(1.60)$ & $t(53)=0.008$ & 0.994 \\
\hline Cho $(I U)$ & $2.45(0.44)$ & $2.54(0.27)$ & $t(55)=-0.928$ & 0.358 \\
\hline $\mathrm{Cr}(\mathrm{IU})$ & $8.40(0.93)$ & $8.30(0.7 I)$ & $t(55)=0.458$ & 0.649 \\
\hline
\end{tabular}

IU: Institutional units.

aNA $n=28$, Glu $n=27$, Glx $n=26$, Cho $n=27$, and $\mathrm{C} r n=27$.

bNAA $n=30$, Glu $n=28$, Glx $n=29$, Cho $n=30$, and $\mathrm{Cr} n=30$.

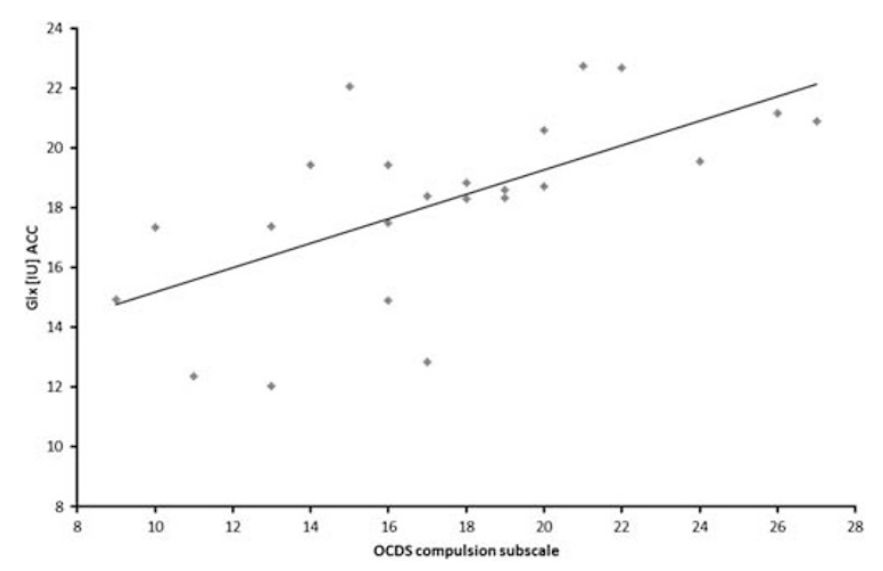

Figure 3 Correlation between the compulsion subscale of the Obsessive Compulsive Drinking Scale (OCDS) and the combined glutamate and glutamine (Glx) level in the anterior cingulate cortex (ACC).

\section{${ }^{1} \mathrm{H}$-MRS of the ACC}

The mean metabolite concentrations in the ACC are displayed in Table 3. There were no significant betweengroup differences with respect to the metabolite concentrations (all $p$-values $>0.05$; Table 3 ). As observed for the NAcc, there were no significant differences between the non-medicated patients and the patients medicated with either diazepam or clomethiazole (all $p$-values $>0.05$ ). In our study, the Glx concentrations in the ACC correlated positively with craving (total OCDS score; $r=0.474$, $p=0.026)$, ie, patients with higher Glx levels in the ACC reported more craving. Again, this positive correlation was noted for the compulsion OCDS subscale $(r=0.628$, $p<0.001$; partial Spearman's $r=0.687, \quad p=<0.001$, Figure 3, controlling for the number of cigarettes smoked per day; partial Spearman's $r=0.702, p<0.001$, controlling for gray matter) but not for the obsession OCDS subscale $(r=0.274, p=0.195)$. Again, separate statistical analysis of estimated Gln concentrations revealed a positive correlation between craving and Gln $(r=0.691, p=0.001$; partial Spearman's $r=0.696, p=0.001$, controlling for the number of cigarettes smoked per day; partial Spearman's $r=0.676$, $p=0.001$, controlling for gray matter). There were no other significant correlations between the metabolite levels and the clinical or demographic characteristics, including the severity of withdrawal symptoms. In patients, there were negative correlations between both the NAA level $(r=-0.410, p=0.037)$ and the Cr level $(r=-0.450$, $p=0.021)$ and the number of cigarettes smoked per day.

\section{DISCUSSION}

To the best of our knowledge, this study is the first to investigate the in vivo Glu levels in the NAcc in alcoholdependent patients. The NAcc is a key region that is implicated in the brain's reward circuit and is regarded as having a crucial function in addiction-related reinforcing mechanisms. The integration of glutamatergic and dopaminergic neurotransmission is thought to be one of the basic mechanisms underlying reward-related learning in cortico-striatal networks (Spanagel, 2009). Medium spiny neurons, which constitute $>90 \%$ of the core and shell NAcc neurons, are characterized by the combined innervation by glutamatergic afferents from the prefrontal cortex, amygdala, and thalamus and dopaminergic afferents from the VTA (Kelley, 2004). Moreover, the results of several animal studies support the pivotal role of the NAcc in alcohol addiction. Microdialysis studies focused on alcohol-consuming rats have demonstrated that there are significant Glu increases in the ventral striatum associated with seizure activity and hyperexcitability during abstinence (Rossetti et al, 1999; Dahchour and De Witte, 2000). Chronic alcohol consumption was found to be associated with morphological changes in the dendritic spines of the medium spiny neurons in the NAcc (Zhou et al, 2007), and metabotropic Glu receptor activity in the NAcc was shown to be required for the maintenance of ethanol self-administration in alcohol-preferring rats (Besheer et al, 2010). Interestingly, in a very recent human off-label investigation in a patient with chronic alcoholism, craving and relapses were reduced by deep brain stimulation in the NAcc (Heldmann et al, 2012). This finding supports the involvement of the ventral striatum in the long-term course of alcohol dependence.

We are unable to draw conclusions from our data concerning the long-term course of glutamatergic dysregulation in the NAcc; however, glutamatergic metabolism in the NAcc was also correlated with craving as measured using the OCDS in our patients. Craving has consistently been shown to be predictive of long-term outcomes and relapse (Schmidt et al, 2011; Heilig et al, 2010; Bottlender et al, 2004). The positive correlation between craving and Glx levels in the NAcc suggests that changes in 
Glu/Gln cycle homeostasis are not related to withdrawal alone. Glutamatergic imbalances within cortico-striatal circuits have previously been hypothesized to underlie the difficulties in modulating drug-seeking behavior (Kalivas and Volkow, 2011). Glu is thought to be the major component of the Glx signal; thus, Glx levels are very likely related to excitatory neurotransmission by, eg, the replenishment of synaptic vesicles as part of the Glu/ Gln cycle. Glu in neuronal synaptic vesicles is the primary source of extracellular Glu, it is released from the vesicles into the synaptic cleft, which results in excitatory neurotransmission, and is then rapidly cleared via high-affinity aminoacid transporters on neurons and astrocytes. To prevent excitotoxicity, these transporters have the capacity to reduce the extracellular Glu concentration quite rapidly to nanomolar levels (Moussawi et al, 2011). In astrocytes, Glu is metabolized into Gln and then transferred back into neurons, where it is converted into Glu and used to refill the synaptic vesicles. Spectroscopy cannot differentiate between these various Glu pools; however, the Glu and Glx signals detected by ${ }^{1} \mathrm{H}$ spectroscopy are very closely related to the results for Glu/ Gln cycling investigated by ${ }^{13} \mathrm{C}$ MRS (Rothman et al, 2011).

As we did not quantify Gln separately by MRS, we estimated Gln levels by subtracting Glu from Glx in both brain areas. These Gln levels were significantly correlated with craving, supporting the alternate hypothesis that the correlation between Glx and craving is mostly driven by Gln. Interestingly, Thoma et al (2011) reported increased levels of Gln in patients with alcohol use disorder, which positively correlated with the long-term severity of alcohol abuse. Still, the limited resolution of both overlapping metabolites with the applied spectroscopy and the complexity of the Glu/Gln cycle as described above allow only cautious interpretations of the differential changes of Glu and Gln.

The Glx levels in the NAcc and the ACC were both strongly correlated with the compulsion OCDS subscale, which comprises the behavioral aspects of alcohol craving. Interestingly, in a study that investigated Glu concentrations in patients with borderline personality disorder, a positive correlation between impulsivity and Glu level in the ACC was reported (Hoerst et al, 2010). The ACC has an important role in the adaptation of behavior, and the rostral ACC is primarily involved in response inhibition related to the affective appraisal of decision making. With regard to behavior, response inhibition is crucial not only for impulsivity in general but also for successful abstinence by individuals with alcohol dependence, eg, in response to alcohol-related cues.

We found no significant differences in the Glu levels in the ACC between patients and controls. This result might be due to the faster normalization of glutamatergic imbalances in the ACC than in the NAcc, which would be consistent with recent data from the study by Hermann et al (2012). These researchers reported increased levels of Glu in the ACC before detoxification, which then normalized within 14 days. Faster normalization in the ACC would also be consistent with the data from the study by Lee et al (2007), who observed no significant differences in the Glu levels between patients and controls after 2 weeks of abstinence. Umhau et al (2010) did not include a control group in their study, so it is difficult to compare their results with our data. These authors reported a decrease in the Glu level in an acamprosate-treated group after 4 weeks, a result that they interpreted as the long-term effect of acamprosate on craving.

There were no other significant differences in metabolite concentrations in our study. In particular, we were unable to find the hypothesized NAA reduction in the ACC, perhaps because the neuronal dysfunction in alcoholdependent patients is a time-limited phenomenon that may be resolved by fast regenerative processes. This hypothesis is supported by animal data from studies by Hermann et al (2012) and Zahr et al (2010), who observed decreased NAA levels in intoxicated animals followed by rapid normalization during abstinence.

There are some limitations to our study. First, we were unable to differentiate between smoking and non-smoking alcohol-dependent patients. As in most studies on alcoholism, the majority of patients were smokers, whereas only a few healthy subjects smoked. We found no correlation between the number of cigarettes smoked and Glu levels in either brain region in the patient group. Also, the results remained stable when the number of cigarettes smoked per day was controlled for. Hence, group differences in Glu levels in the NAcc should not be caused by smoking habits. Nevertheless, some animal studies support the hypothesis that the administration of nicotine increases Glu in various brain regions (Gass and Olive, 2008); however, these results are controversial. Wang et al (2007) reported the upregulation of ionotropic Glu subunits in the prefrontal cortex and the VTA but not in the NAcc in chronically nicotine selfadministering rats. Lallemand et al (2011) even observed diminished Glu release in the NAcc with the co-administration of nicotine in binge-drinking rats. Spectroscopy studies investigating differences between subjects who smoke and those who do not have found either no differences or reduced levels of Glu in the ACC in smokers (Mashhoon et al, 2011; Gallinat and Schubert, 2007). In our study, the number of cigarettes smoked by patients was negatively correlated with the NAA level in the ACC. These data are consistent with the results obtained by Durazzo et al (2011), who investigated smoking and non-smoking alcohol-dependent patients and reported decreased cortical thickness in the ACC and lower levels of NAA in the frontal lobes related to smoking.

Second, the majority of patients were investigated after acute detoxification with either diazepam or clomethiazole using a standardized treatment strategy. According to the treatment protocol, the patients with the most distinct withdrawal symptoms received the highest total doses of medication. This finding may explain why we were unable to find any correlation between the severity of withdrawal and the Glu levels. Diazepam and clomethiazole are both GABAergic and are thought to reduce extracellular Glu levels (Khan et al, 1999). Thus, the elevated Glu levels in the NAcc despite medication use during detoxification in patients with severe withdrawal symptoms support our hypothesis of persistent striatal glutamatergic dysfunctions in the NAcc after detoxification. In addition, we found no significant differences in the Glu levels between patients treated with diazepam or clomethiazole and patients not treated with medication, a finding that supports our hypothesis that increased Glu levels are not the result of the medication. In addition, we investigated only male 
alcoholics; thus, our results might not apply to female patients. There is some evidence that there are differential adaptations of GABAergic and glutamatergic neurotransmission between female and male rats during acute withdrawal (Alele and Devaud, 2005). Therefore, future studies should be performed with both sexes.

Finally, although gray matter fractions in both VOIs did not differ between patients and controls, the amount of gray matter had an impact on the within-group correlation between craving and Glx in the NAcc: partial correlations for the gray matter fraction reduced the significance level of the correlation between craving and Glx to $p=0.07$. This now marginally significant correlation still supports our hypothesis of a correlation between craving and the dysregulation of the glutamatergic system in the NAcc, still, future studies with probably more exclusive measurements of the NAcc gray matter should be conducted to confirm these data.

Also, as we optimized our MRS design to detect Glu in a relatively small VOI with low signal-to-noise, we had to accept some drawbacks in the measurements of all other metabolites resulting in higher variances of most metabolite levels. These methodological limitations might also be responsible for some of the differences in metabolite levels compared with previous studies.

In conclusion, our results validate data from animal studies suggesting that upregulated glutamatergic neurotransmission in the NAcc is associated with alcohol withdrawal. Our data also strengthen the hypothesis that glutamatergic dysfunction is involved in craving and relapse and, thus, in the long-term course of alcoholism. Finally, the updated spectroscopic methodology proved to be a reliable and useful approach for investigating the cerebral glutamatergic system in vivo.

\section{ACKNOWLEDGEMENTS}

We are grateful to both the administration (Director: Dr Jutta Settelmayer) and the staff, especially $\mathrm{Dr}$ Thomas Wefelmeyer, of the Department of Addiction, Westphalian Psychiatric Clinics Muenster, for their cooperation.

\section{DISCLOSURE}

The authors declare no conflict of interest.

\section{REFERENCES}

Alele PE, Devaud LL (2005). Differential adaptations in GABAergic and glutamatergic systems during ethanol withdrawal in male and female rats. Alcohol Clin Exp Res 29: 1027-1034.

Anton RF, Moak DH, Latham P (1996). The obsessive compulsive drinking scale. Arch Gen Psychiatry 53: 225-231.

Anton RF, Moak DH, Latham P (1995). The obsessive compulsive drinking scale: a self-rated instrument for the quantification of thoughts about alcohol and drinking behavior. Alcohol Clin Exp Res 19: 92-99.

Besheer J, Grondin JJ, Cannady R, Sharko AC, Faccidomo S, Hodge CW (2010). Metabotropic glutamate receptor 5 activity in the nucleus accumbens is required for the maintenance of ethanol self-administration in a rat genetic model of high alcohol intake. Biol Psychiatry 67: 812-822.
Bottlender M, Soyka M (2004). Impact of craving on alcohol relapse during, and 12 months following, outpatient treatment. Alcohol Alcohol 39: 357-361.

Dahchour A, De Witte P (2000). Taurine blocks the glutamate increase in the nucleus accumbens microdialysate of ethanoldependent rats. Pharmacol Biochem Behav 65: 345-350.

De Witte P (2004). Imbalance between neuroexcitatory and neuroinhibitory amino acids causes craving for ethanol. Addict Behav 29: 1325-1339.

Durazzo TC, Mon A, Gazdzinski S, Meyerhoff DJ (2011). Chronic cigarette smoking in alcohol dependence: associations with cortical thickness and $\mathrm{N}$-acetylaspartate levels in the extended brain reward system. Addict Biol Epub.

Ende G, Welzel H, Walter S, Weber-Fahr W, Diehl A, Hermann D et al (2005). Monitoring the effects of chronic alcohol consumption and abstinence on brain metabolism: a longitudinal proton magnetic resonance spectroscopy study. Biol Psychiatry 58: 974-980.

Fortier CB, Leritz EC, Salat DH, Venne JR, Maksimovskiy AL, Williams $\mathrm{V}$ et al (2011). Reduced cortical thickness in abstinent alcoholics and association with alcoholic behavior. Alcohol Clin Exp Res 35: 2193-2201.

Gallinat J, Schubert F (2007). Regional cerebral glutamate concentrations and chronic tobacco consumption. Pharmacopsychiatry 40: 64-67.

Gass JT, Olive MF (2008). Glutamatergic substrates of drug addiction and alcoholism. Biochem Pharmacol 75: 218-265.

Groenewegen HJ, Wright CI, Beijer AV, Voorn P (1999). Convergence and segregation of ventral striatal inputs and outputs. Ann N Y Acad Sci 877: 49-63.

Gu M, Zahr NM, Spielman DM, Sullivan EV, Pfefferbaum A, Mayer D (2012). Quantification of glutamate and glutamine using constant-time point-resolved spectroscopy at $3 \mathrm{~T}$. NMR Biomed 26: $164-172$.

Gsellhofer B, Fahrner EM, Platt JJ (1994). Manual für Training und Durchführung von Interviews mit dem EuropASI. München, IFT: German version.

Hancu I (2009). Optimized glutamate detection at 3T. J Magn Reson Imaging 30: 1155-1162.

Heilig M, Egli M, Crabbe JC, Becker HC (2010). Acute withdrawal, protracted abstinence and negative affect in alcoholism: Are they linked? Addict Biol 15: 169-184.

Heldmann M, Berding G, Voges J, Bogerts B, Galazky I, Müller U et al (2012). Deep brain stimulation of nucleus accumbens region in alcoholism affects reward processing. PLoS One 7: e36572.

Hermann D, Weber-Fahr W, Sartorius A, Hoerst M, Frischknecht U, Tunc-Skarka N et al (2012). Translational magnetic resonance spectroscopy reveals excessive central glutamate levels during alcohol withdrawal in humans and rats. Biol Psychiatry 71: 1015-1021.

Hinton DJ, Lee MR, Jacobson TL, Mishra PK, Frye MA, Mrazek DA et al (2012). Ethanol withdrawal-induced brain metabolites and the pharmacological effects of acamprosate in mice lacking ENT1. Neuropharmacology 62: 2480-2488.

Hoerst M, Weber-Fahr W, Tunc-Skarka N, Ruf M, Bohus M, Schmahl C et al (2010). Correlation of glutamate levels in the anterior cingulate cortex with self-reported impulsivity in patients with borderline personality disorder and healthy controls. Arch Gen Psychiatry 67: 946-954.

Kalivas PW, Volkow ND (2011). New medication for drug addiction hiding in glutamatergic neuroplasticity. Mol Psychiatry 16: 974-986.

Kelley AE (2004). Memory and addiction: shared neural circuitry and molecular mechanisms. Neuron 44: 161-179.

Khan GM, Smolder I, Lindekens H, Manil J, Ebinger G, Michotte Y (1999). Effects of diazepam on extracellular brain neurotransmitters in pilocarpine-induced seizures in rats. Eur J Pharmacol 373: $153-161$. 
Krupitsky EM, Rudenko AA, Burakov AM, Slavina TY, Grinenko AA, Pittman B et al (2007). Antiglutamatergic strategies for ethanol detoxification: comparison with placebo and diazepam. Alcohol Clin Exp Res 31: 604-611.

Lallemand F, Ward RJ, De Witte P, Verbanck P (2011). Binge drinking $+I-$ chronic nicotine administration alters extracellular glutamate and arginine levels in the nucleus accumbens of adult male and female Wistar rats. Alcohol Alcohol 46: 373-382.

Lee E, Jang DP, Kim JJ, An SK, Park S, Kim IY et al (2007). Alteration of brain metabolites in young alcoholics without structural changes. Neuroreport 18: 1511-1514.

Licata SC, Renshaw PF (2010). Neurochemistry of drug action: insights from proton magnetic resonance spectroscopic imaging and their relevance to addiction. Ann N Y Acad Sci 1187: 148-171.

Mashhoon Y, Janes AC, Jensen JE, Prescot AP, Pachas G, Renshaw $\mathrm{PF}$ et al (2011). Anterior cingulate proton spectroscopy glutamate levels differ as a function of smoking cessation outcome. Neuropsychopharmacol Biol Psychiatry 35: 1709-1713.

Mason G, Bendszus M, Meyerhoff D, Hetherington H, Schweinsburg B, Ross B et al (2005). Magnetic resonance spectroscopic studies of alcoholism: from heavy drinking to alcohol dependence and back again. Alcohol Clin Exp Res 29: 150-158.

Miller GA, Chapman JP (2001). Misunderstanding analysis of covariance. J Abnorm Psychol 110: 40-48.

Modi S, Bhattacharya M, Kumar P, Deshpande SN, Tripathi RP, Khushu S (2011). Brain metabolite changes in alcoholism: localized proton magnetic resonance spectroscopy study of the occipital lobe. Eur J Radiol 79: 96-100.

Moussawi K, Riegel A, Nair S, Kalivas PW (2011). Extracellular glutamate: functional compartments operate in different concentration ranges. Front Syst Neurosci 5: 94.

Mullins PG, Chen H, Caprihan A, Gasparovic C (2008). Comparative reliability of proton spectroscopy techniques designed to improve detection of J-coupled metabolites. Magn Res Med 60: 964-969.

Mundle G, Banger M, Mugele B, Stetter F, Soyka M, Veltrup C et al (2006). Alkoholbezogene Störungen-Akutbehandlung. In: Schmidt LGGastpar MFalkai PGaebel W (eds) Evidenzbasierte Suchtmedizin. Deutscher Ärzte-Verlag: Köln, pp 25-52.

Omelchenko N, Sesack SR (2007). Glutamate synaptic inputs to ventral tegmental area neurons in the rat derive primarily from subcortical sources. Neuroscience 146: 1259-1274.

Provencher SW (1993). Estimate of metabolite concentrations from localized in vivo proton NMR spectra. Magn. Reson. Med 30: 672-679.

Roesner S, Leucht S, Lehert P, Soyka M (2008). Acamprosate supports abstinence, naltrexone prevents excessive drinking: evidence from a meta-analysis with unreported outcomes. J Psychopharmacol 22: 11-23.
Rothman DL, De Feyter HM, de Graaf RA, Mason GF, Behar KL (2011). ${ }^{13} \mathrm{C}$ MRS studies of neuroenergetics and neurotransmitter cycling in humans. NMR Biomed 24: 943-957.

Rossetti ZL, Carboni S, Fadda F (1999). Glutamate-induced increase of extracellular glutamate through $N$-methyl-D-aspartate receptors in ethanol withdrawal. Neuroscience 93: 1135-1140.

Saunders JB, Aasland OG, Babor TF, de la Fuente JR, Grant M (1993). Development of the Alcohol Use Disorders Screening Test (AUDIT). WHO collaborative project on early detection of persons with harmful alcohol consumption. II. Addiction 88: 791-804.

Schmidt P, Helten C, Soyka M (2011). Predictive value of obsessive-compulsive drinking scale (OCDS) for outcome in alcohol-dependent inpatients: results of a 24-month follow-up study. Subst Abuse Treat Prev Policy 6: 14.

Siggins GR, Martin G, Roberto M, Nie Z, Madamba S, De Lecea L (2003). Glutamatergic transmission in opiate and alcohol dependence. Ann N Y Acad Sci 1003: 196-211.

Spanagel R (2009). Alcoholism: a systems approach from molecular physiology to addictive behavior. Physiol Rev 89: 649-705.

Thoma R, Mullins P, Ruhl D, Monnig M, Yeo RA, Caprihan A et al (2011). Perturbation of the glutamate-glutamine system in alcohol dependence and remission. Neuropsycho-pharmacology 36: 1359-1365.

Umhau JC, Momenan R, Schwandt ML, Singley E, Lifshitz M, Doty $\mathrm{L}$ et al (2010). Effect of acamprosate on magnetic resonance spectroscopy measures of central glutamate in detoxified alcohol-dependent individuals: a randomized controlled experimental medicine study. Arch Gen Psychiatry 67: 1069-1077.

Wang F, Chen H, Steketee JD, Sharp BM (2007). Upregulation of ionotropic glutamate receptor subunits within specific mesocorticolimbic regions during chronic nicotine self-administration. Neuropsychopharmacology 32: 103-109.

Ward RJ, Lallemand F, De Witte P (2009). Biochemical and neurotransmitter changes implicated in alcohol-induced brain damage in chronic or 'binge drinking' alcohol abuse. Alcohol Alcohol 44: 128-135.

Wijtenburg AS, Knight-Scott J (2011). Very short echo time improves the precision of glutamate detection at $3 \mathrm{~T}$ in ${ }^{1} \mathrm{H}$ Magnetic resonance spectroscopy. J Magnetic Resonance Imaging 34: 645-652.

Zahr NM, Mayer D, Rohlfing T, Hasak MP, Hsu O, Vinco S et al (2010). Brain injury and recovery following binge ethanol: evidence from in vivo magnetic resonance spectroscopy. Biol Psychiatry 67: 846-854.

Zhou FC, Anthony B, Dunn KW, Lindquist WB, Xu ZC, Deng P (2007). Chronic alcohol drinking alters neuronal dendritic spines in the brain reward center nucleus accumbens. Brain Res 1134: 148-161. 